\title{
APPLICATION OF ARTIFICIAL INTELLIGENCE (AI) PROGRAMMING TECHNIQUES TO TACTICAL GUIDANCE FOR FIGHTER AIRCRAFT
}

\author{
John W. McManus \\ and \\ Kenneth H. Goodrich \\ NASA Langley Research Center \\ Mail Stop 489 \\ Hampton, Virginia 23665-5225 \\ (804)864-4037/(804)864-4009
}

\author{
AIAA Guidance, Navigation, and Control Conference \\ August 14-16, 1989 \\ Boston, Massachusetts \\ Autonomous Systems and Mission Planning
}

\begin{abstract}
A research program investigating the use of Artificial Intelligence (AI) techniques to aid in the development of a Tactical Decision Generator (TDG) for Within-Visual-Range (WVR) air combat engagements is discussed. The application of AI methods for development and implementation of the TDG is presented. The history of the Adaptive Maneuvering Logic (AML) program is traced and current versions of the AML program are compared and contrasted with the TDG system. The Knowledge-Based Systems (KBS) used by the TDG to aid in the decision-making process are outlined in detail and example rules are presented. The results of tests to evaluate the performance of the TDG versus a version of AML and versus human pilots in the Langley Differential Maneuvering Simulator (DMS) are presented. To date, these results have shown significant performance gains in one-versus-one air combat engagements, and the AI-based TDG software has proven to be much easier to modify than the updated FORTRAN AML programs.
\end{abstract}

\section{INTRODUCTION}

The development of all-aspect and "fire and forget" weapons has increased the complexity of the air-to-air combat environment. Modern sensors provide critical tactical information to the aircraft a range and precision that were impossible 20 years ago. This increased complexity, combined with the expanded capabilities of high performance aircraft, has changed the future of air combat engagements. The need for a modern, realistic air combat simulation that can be used to evaluate the current and future air combat environment has been well documented [Burgin 1975, 1986, 1988; Hankins 1979]. Existing tools such as the Adaptive Maneuvering Logic program (AML) [Burgin 1975, 1986, 1988], TAC Brawler [Kerchner 1985], and AASPEM have generally centered their efforts on the development and refinement of high-fidelity aircraft dynamics modeling techniques and not on the development 
and refinement of tactical decision generation logic for WVR engagements. In support of the study of superagile aircraft at Langley Research Center (LaRC) a Tactical Guidance Research and Evaluation System (TGRES, pronounced "tigress") is being developed [Goodrich 1989].

\section{Figure 1. TGRES SYSTEM.}

\section{TGRES DESCRIPTION}

The TGRES system, shown in figure 1, provides a means by which researchers can develop and evaluate, in a tactically significant environment, various systems for high performance aircraft. While TGRES is aimed specifically at the development and evaluation of maneuvering strategies and advanced guidance/control systems for superagile aircraft, TGRES's modularity will make it easily adaptable to the analysis of other types of aircraft systems. TGRES is composed of three main elements--the TDG, the Tactical Maneuver Simulator (TMS), and the DMS.

The TDG is a knowledge-based guidance system designed to provide insight into the tactical benefits and costs of enhanced aircraft controllability and maneuverability throughout an expanded flight envelope (i.e. superagility). The two remaining elements of TGRES, the TMS and the DMS, provide simulation environments in which the TDG is exercised. The TMS simulation environment was developed using conventional computer languages on a VAXStation 3200. The TDG was developed on a Symbolics 3650 workstation. The separation of the aircraft simulation and decision logic components allows each module to be developed using hardware and programming techniques specifically designed for its function. This separation of tasks also increases the efficiency of the simulation by allowing some parallel processing. The two processes are executed as co-tasks and communicate via an EtherNet connection. (See fig. 2.)

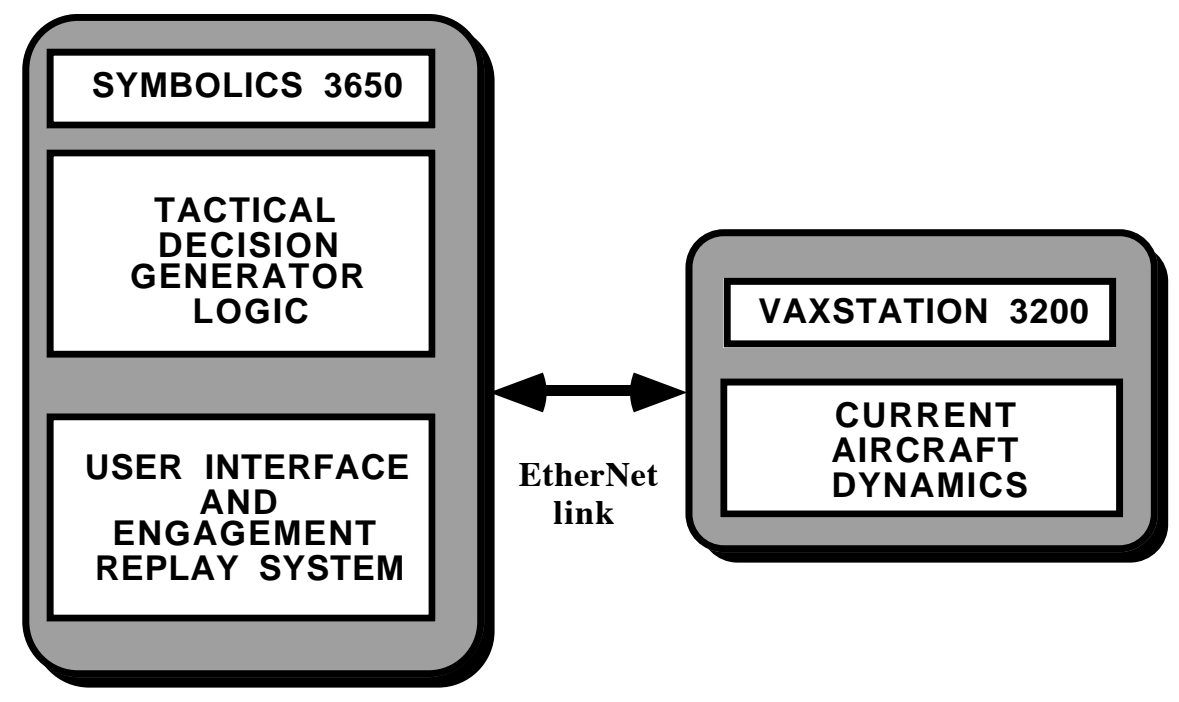




\section{Figure 2. CURRENT HARDWARE CONFIGURATION.}

The user interface system consists of a color graphics package designed to replay both TMS and DMS engagements, and a mouse sensitive representation of the TDG aircraft and its basic systems that allows the user to interact with the TDG aircraft during the execution of TMS runs. The Engagement Replay System (ERS) software is available for a VAX color workstation and a Symbolics color workstation. The ERS display, shown in figure 3, displays the two aircraft on a three-dimensional axis and has dedicated windows used to display several aircraft variables including the thrust, Mach number, and deviation angles of the two aircraft. The viewing angle for each engagement can be rotated $360^{\circ}$ around both the $\mathrm{X}$ and $\mathrm{Z}$ axis to provide the most information to the user. The interactive TMS display includes a graphical representation of the TDG aircraft's major systems such as engines, offensive and defensive systems, and a system status display. During the simulation run the user can enable and/or disable the aircraft's systems using the mouse sensitive display and evaluate how the changes effect the TDG's decision generation process.

\section{Figure 3. ERS DISPLAY.}

The final element of TGRES is the Differential Maneuvering Simulator. The DMS consists of two 40' diameter domes located at Langley. The facility is intended for the real-time simulation of engagements between piloted aircraft. By using the TDG to drive one of the airplanes, it is possible to test the TDG against a human opponent. This feature allows the guidance logic to be evaluated against an unpredictable and adaptive opponent. A third dome (20' in diameter) is being added to the DMS facility. This addition will allow the guidance logic to be evaluated in one-versus-two or two-versus-one scenarios, further enhancing the tactical capability of the DMS environment.

\section{THE AML PROGRAM}

The TDG is being developed as a KBS incorporating some of the features first outlined in the AML program [Burgin 1975, Hankins 1979]. The AML program was selected as a baseline for several reasons, including its past performance as a real-time WVR tactical adversary in the Langley DMS and the modular design of the FORTRAN source code. The tactical decision generation method developed for the original AML program, outlined in figure 4 , is a unique approach that attempts to model the goal-seeking behavior of a pilot by mapping the physical situation between the two aircraft into a finite, abstract situation space. A set of the three basic control variables (bank angle, load factor, and thrust) can be determined to maximize some performance index in the situation space [Burgin 76]. Each triplet of control 
variables defines an "elemental maneuver," and a sequence of these elemental maneuvers may form classical or "text book" air combat maneuvers.

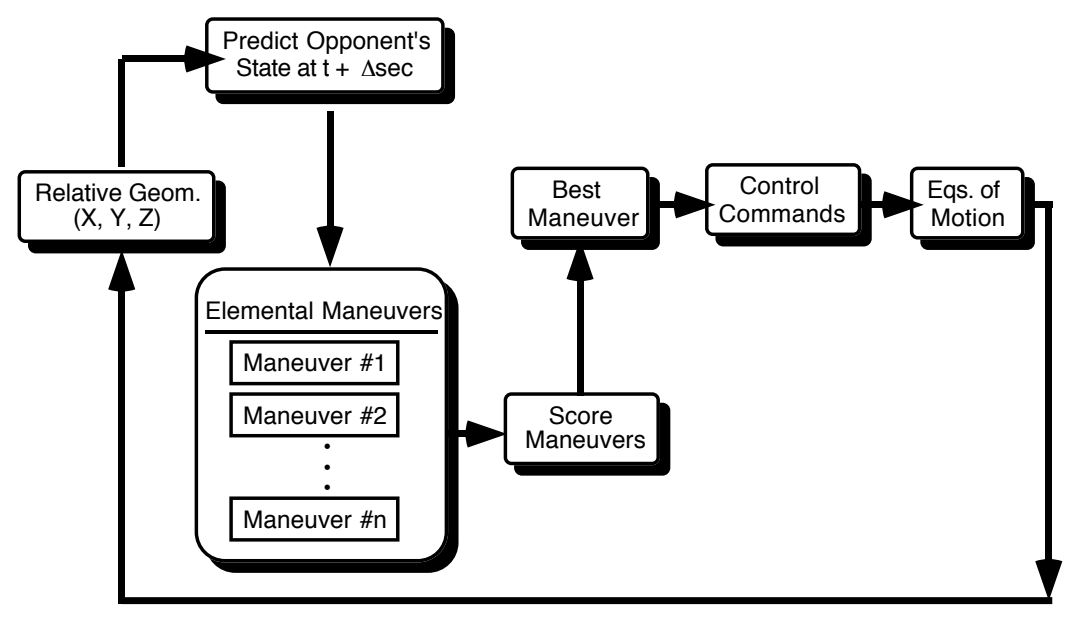

Figure 4. HOW AML WORKS.

Although the logic and geometry used by AML to make tactical decisions is complex, the basic concepts it uses are simple. At each decision interval, the "attacking" aircraft predicts the future position and velocity of its opponent using a curve-fitting algorithm and past known positions of the opponent. The attacker then uses a set of elemental maneuvers (described above) to predict a set of positions that it can reach from its current state.

The AML program forms a "situation state vector" for each trial maneuver evaluated. The vector is used to represent the responses to a set of questions about the current situation. Figure 5 shows the binary scoring method $(0=\mathrm{NO}, 1=\mathrm{YES})$ used to determine the value of each each cell in the vector.

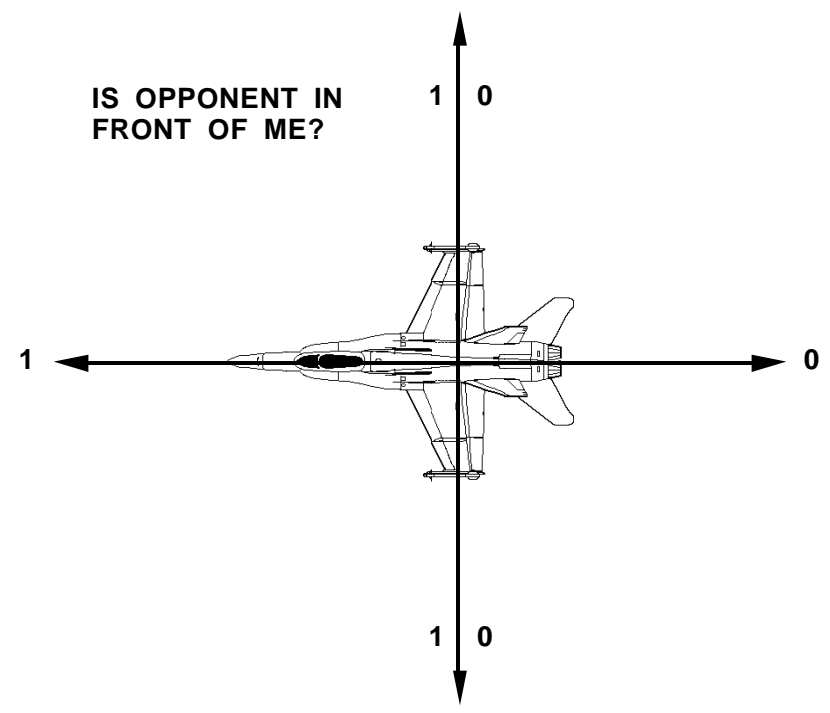


This vector is multiplied by a "scoring weight" vector to form a scalar product that represents the situation space value for the current maneuver. A detailed description of the trial maneuver generation and scoring process and an explanation of how the scoring weights have evolved can be found in [Burgin 1988]. The questions used to form the situation state vector were obtained from several sources including air combat maneuvering manuals, interviews with fighter pilots, and detailed analysis of the original DMS engagements. In the original version of AML, each question had a positive, non-zero weight. The questions were formulated so that a "YES" answer reflects a favorable condition, increasing the score for the maneuver. It is important to note that in the original AML research "no systematic investigation was made to optimize these weight factors; they were usually all set to one." The early AML versions [Burgin 1975; Hankins 1979] were designed to perform as a conservative opponent. The scoring rules rated offense and defense evenly and risked giving up some positional advantage to the opponent only when there was reasonable assurance the attacker would gain at least as much offsetting advantage. This conservative approach may be the product of a philosophy stated in [Burgin 1988],

\footnotetext{
"The objective of the decision-making process is to derive maneuvers which will bring one's own weapons to bear on the target while at the same time minimizing exposure to the other side's weapons."
}

This is a one-dimensional approach to the problem. It outlines a logic that handles only the neutral and aggressive cases effectively and does not recognize that there are several Modes of Operation (MO), outlined in figure 6, that a pilot may use during an engagement. In many situations when the opponent has a distinct positional advantage, the AML aircraft will perform "kamikaze" maneuvers, giving up one or more clear shots to the opponent while it maneuvers to a position of "advantage." In these situations the AML aircraft would not survive to exploit the positional advantage, having been "killed" while obtaining it.

- AGGRESSIVE

- DEFENSIVE

- NEUTRAL

- EVASIVE

- EVADING OPPONENTS' "LOCK AND FIRE"

- EVADING MISSILE (AAM \& SAM)

- GROUND / STALL EVASION

Figure 6. TDG MODES OF OPERATION. 
The existing trial maneuver versions of AML do a good job of getting behind an opponent, but due to the grain of the trial maneuvers, lack the ability to fine-track the opponent. Several changes were made to the AML program [Burgin 1988] to address this problem. The requirement that only the opponents positional data be passed to the algorithm was relaxed and "complete and accurate information about the the opponent's past and present states" is now provided. The 1986 version of the AML program, AML86 [Burgin 1988], also made several major changes to the tactical decision generation process, abandoning the trial maneuver concept for a rule-based approach and a set of canned "Basic Fighter Maneuvers." [Burgin 1988] contains an extensive history of the "trial maneuver" concept and a description of how the new rule-based version of the program, AML68, was developed. A "pointing" control system was also developed to aid the fine-tracking process. The pointing control system directly commands roll and pitch rates to point the aircraft's longitudinal axis at the opponent. AML86 is a first step towards a multi-dimensional approach and is similar to the decision logic incorporated in the TDG.

\section{THE DEVELOPMENT OF THE TDG SYSTEM}

The development of the TDG has been a multi-stage process using the COSMIC version of AML as a starting point. The COSMIC version of AML was updated by Dynamics Engineering Incorporated (DEI) while under contract to NASA Langley. This version of AML, (DEI-AML), has a scoring module that uses a set of 15 binary questions and a fixed set of weights to evaluate the trial maneuvers. DEI installed aerodynamic data and engine characteristics provided by the Aircraft Guidance and Controls Branch (AGCB) into the AML data tables and made all changes to the AML software outlined in [Burgin, 1986]. DEI-AML was tested by AGCB to insure symmetry of the engagements given symmetric initial conditions. During the testing process several software bugs were found and corrected. A full description of the bugs and corrections are outlined in [McManus 1989]. The resulting code, dubbed AML', was again tested for symmetry and a DMS ready version of the code, DMS$\mathrm{AML}^{\prime}$ was prepared. $\mathrm{AML}^{\prime}$ and a DMS ready version, DMS-AML', are being used as the baseline during development of the TDG system. 


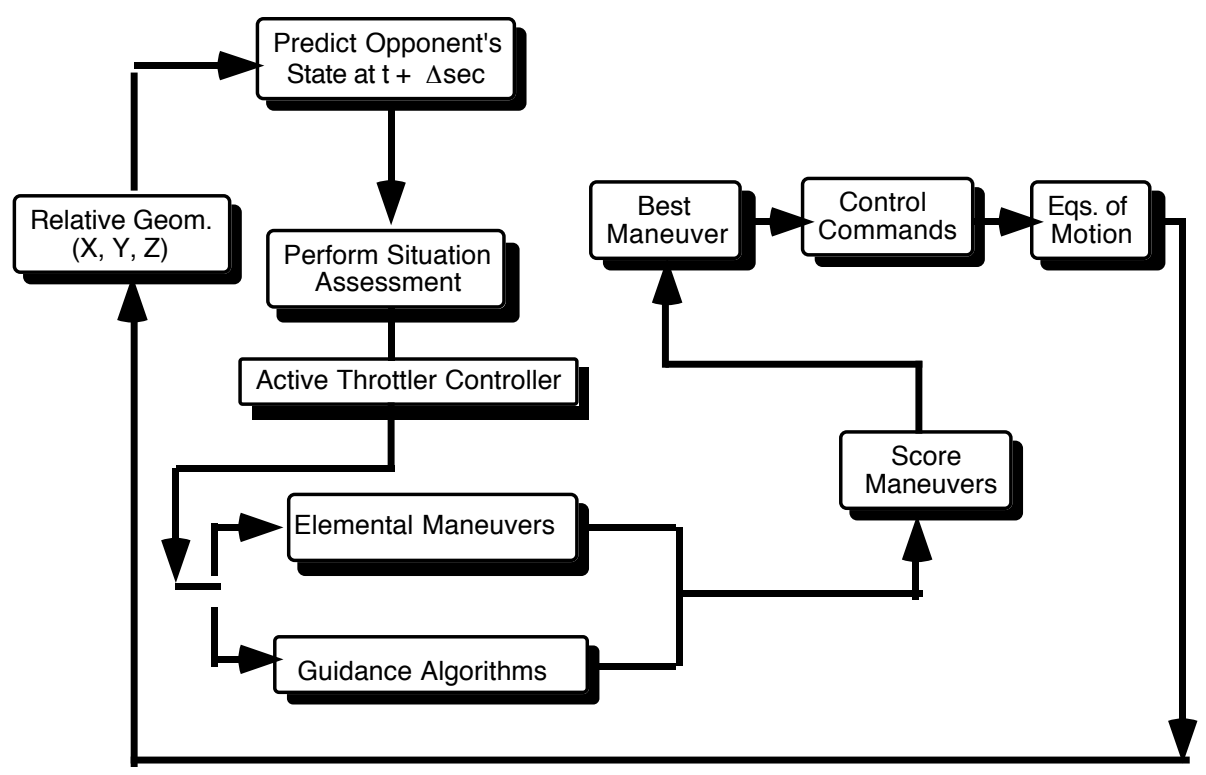

Figure 7. HOW TDG WORKS.

The TDG system, outlined in figure 7, currently uses the trial maneuver concept outlined in the AML program with several extensions. The original set of five to nine trial maneuvers has been expanded to include over 40 trial maneuvers. Although this is a "brute force" solution the new trial maneuvers allow the TDG to perform target tracking more effectively and improve the system's overall performance. The TDG uses an object-oriented programming approach to represent each aircraft and the current state of its offensive systems, defensive systems, and engines. This information is used to help guide the TDG's reasoning process. The original FORTRAN AML throttle controller and the maneuver scoring modules have been redesigned using a rule-based programming approach and ported to the AI workstation. Examples of rules for each of the KBS modules are shown in figure 8. 


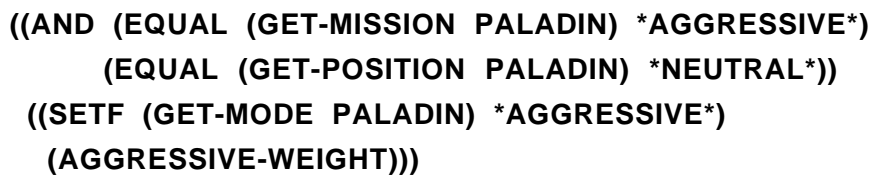

EXAMPLE MODE SELECTION RULE.

((AND I-SEE-HIM I-CAN-FIRE HE-CANT-FIRE (<= RANGE 12500))

EXAMPLE THROTTLE CONTROL RULE.

((OR ( $\leq$ (ABS HIM-UNABLE-TO-FIRE) (I-CAN-FIRE))

(AND (> HIM-UNABLE-TO-FIRE O.0) ( $\geq$ I-CAN-FIRE 0.0) )

(= GUNA 1.0)

(= ALLA 1.0)

(= HEATA 1.0))

(SETF (GET-POSITION PALADIN) *AGGRESSIVE*))

EXAMPLE SITUATION ASSESSMENT RULE.

Figure 8. Example Rules.

\section{KBS MODULES OF THE TDG}

The TDG system has a knowledge-based Situation Assessment (SA) module that is executed at each decision interval before the trial maneuvers are evaluated. The SA module is used to determine the TDG's current MO. The SA is executed at each interval, before the maneuver scoring module, and determines the TDG's MO. This determination is based on the TDG's current mission, the current state of the aircraft's systems, the relative geometry between the aircraft and its opponent, and the opponent's instantaneous-intent (*in-int*). Each of the modes shown in figure 6 has a unique set of scoring weights and a decision interval associated with it. The weights for each mode have been adjusted during the design and testing process to maximize the TDG's performance in that mode. Test results have shown that a short decision interval, $(0.5 \mathrm{sec}$.), improves the TDG's fine-tracking performance. The same short decision interval results in a "thrashing" motion in neutral situations resulting in degraded system performance. A longer decision interval, $(1.0 \mathrm{sec}$.), is used in neutral situations. The opponent's *in-int* is defined to be an estimation of the opponent's intent at the current point in time based on available sensor, positional, and geometric data. Currently, there is no attempt to use a history of *in-int* to derive a long-term opponent intent. The flexibility provided by the use of MO's allows the system to more closely model the pilots changing strategies during the engagement. The COSMIC version of AML, and most AML variations before AML86, do not have the ability to change their decision generation strategy based on the changing environment. The TDG Scoring Module (SM) is a KBS that uses a set of 17 fuzzy logic 
questions with responses ranging from $[0=\mathrm{NO}, \ldots, 1.0=\mathrm{YES}]$, (fig. 8), and the set of mode-specific scoring weights selected by the SA module to score each of the trial maneuvers. A rule-based active Throttle Controller (TC) has been developed to replace the existing throttle control subroutine. The TC is called at the start of each decision interval and can set the throttle at any position from idle to full afterburner $[0, \ldots, 2]$. The logic for the existing AML throttle control subroutine had only three positions $(0=$ idle, $1=$ military, $2=$ full afterburner $)$ and had been turned off in the COSMIC version--all engagements were being flown with the throttle set at full afterburner.

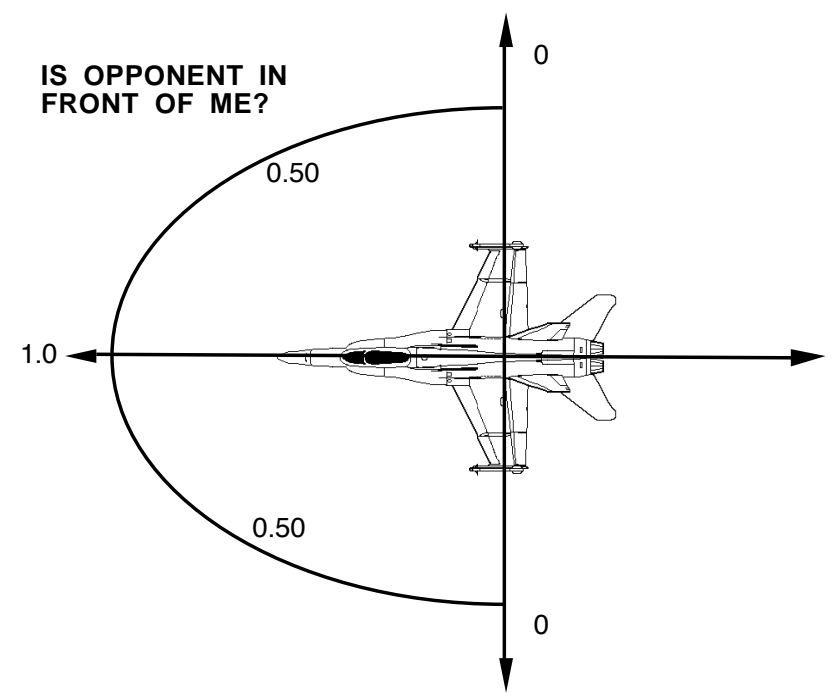

Figure 9. EXAMPLE OF TDG SCORING.

\section{TDG TESTING PROCEDURES}

The TDG is currently being tested in the laboratory and in the LaRC DMS using the existing AML aircraft dynamics. Laboratory testing is done in a non-real-time, batch mode environment against the AML' baseline. Each set of tests consists of 64 sets of initial aircraft conditions as shown in figure 9 . Both airplanes have a heading of 0 degrees in the first run. The TDG's heading is rotated 45 degrees in each subsequent engagement $(0,45,90, \ldots)$. For each complete rotation of the TDG airplane AML s rotated 45 degrees. 


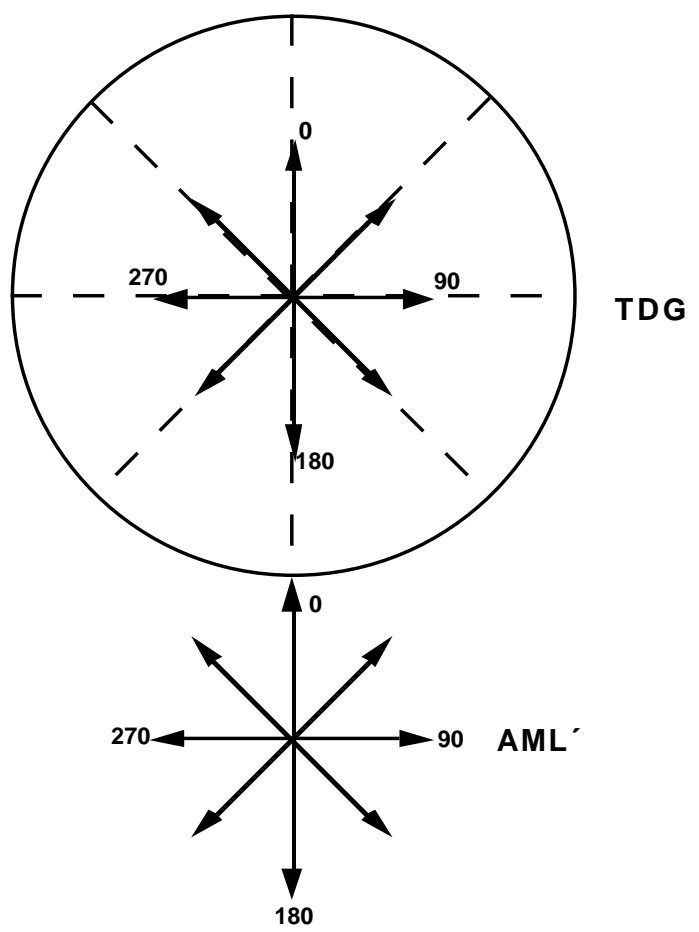

Figure 10. SET OF 64 INITIAL CONDITIONS.

A statistics module is used to calculate the amount of time that each aircraft has its weapons locked on its opponent and the deviation angle and angle-off. The Line-Of-Sight (LOS) vector is defined as the vector between ownship c.g. and opponent's c.g. The Line-Of-Sight (LOS) angle is defined as the angle between the LOS vector and ownship body $\mathrm{x}$-axis; the deviation angle is defined as the angle between the LOS vector and ownship velocity vector; and the angle-off is defined as the angle between the LOS vector and opponent's velocity vector (fig. $10)$. 


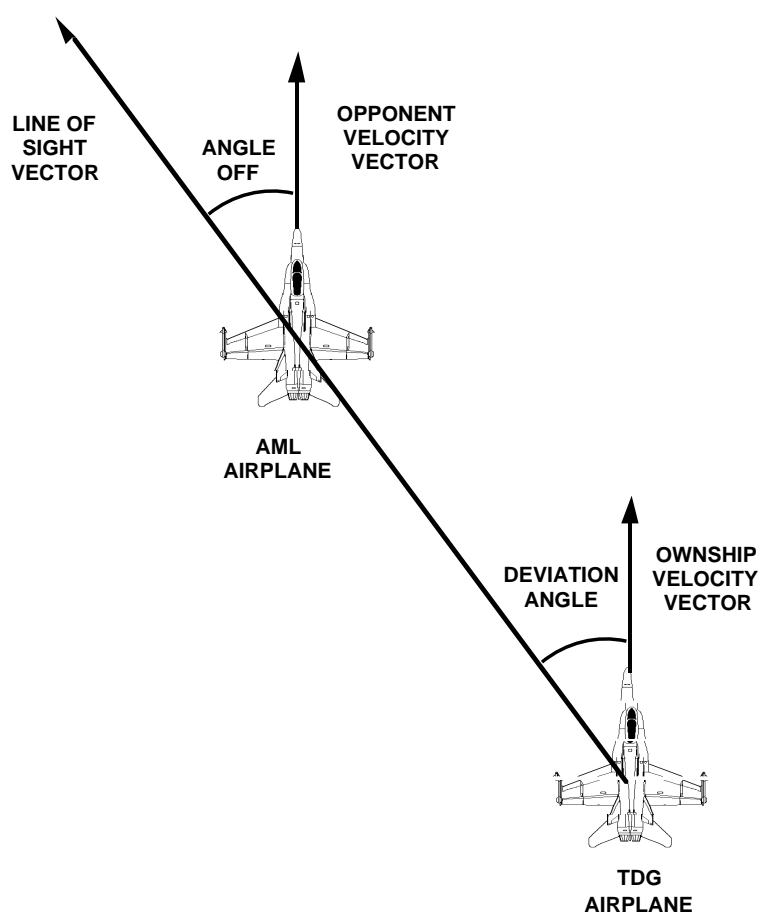

Figure 11. ANGLE DEFINITIONS.

The weapons cones used represent a generic all-aspect missile, a generic tail-aspect missile, and a $20 \mathrm{~mm}$ cannon (fig. 11). Four metrics are currently used to evaluate each engagement. The first metric is calculated every second and computes the total time that each airplane has its weapons locked on the opponent, the probability that the shot will hit, the distance between the opponents, the angle-off, and the deviation angle. The results are printed in a table format at the completion of each run. The second metric computes a Probability of Survival (PS) using the data computed by the first metric. The missiles are treated as a limited resource and a probability to hit of 0.65 is required to launch the first missile. The firing threshold increases by 0.05 for each missile launched, and all missiles are required to complete their flight to the target before the next missile is fired. The third scoring metric attempts to determine a Lethal Time (LT) value for each engagement. The LT value for a run is equal to ((TDG gun time AML gun time) / 2) + 2* (TDG tail-aspect time - AML tail-aspect time) + (TDG all-aspect time - AML all-aspect time). A positive LT value shows TDG with an advantage, a negative LT shows AML with an advantage. The fourth metric is Time on Offense (TOF). TOF is the sum of all weapons lock time for each each airplane. $\triangle \mathrm{TOF}$ is computed as TDG TOF minus AML TOF. 


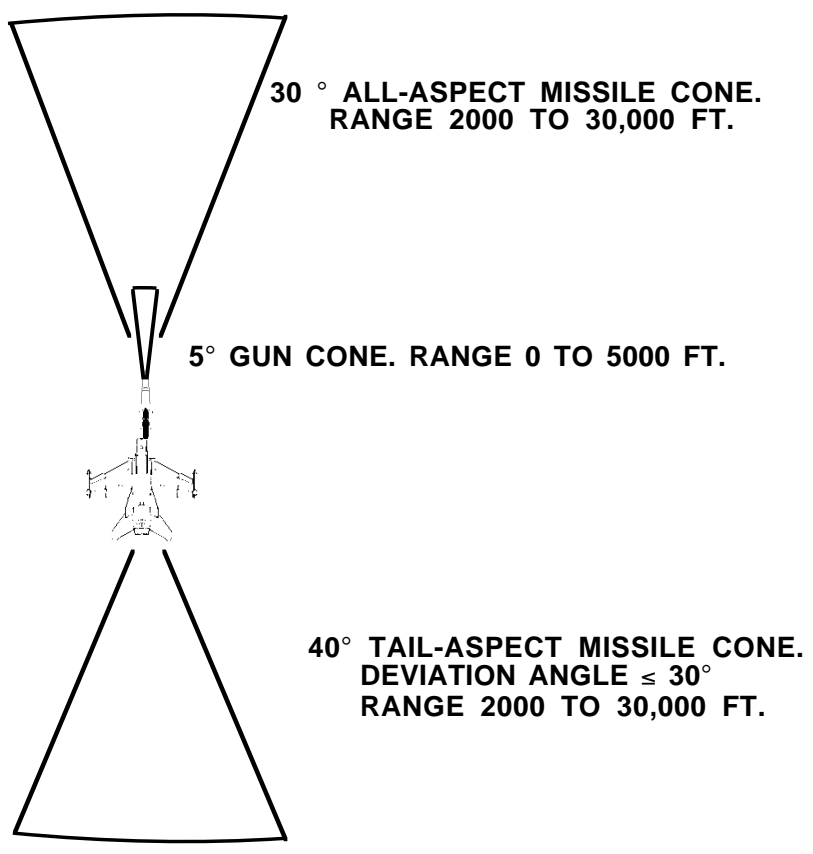

Figure 12. WEAPONS CONES.

These statistics are reviewed after each set of runs and the data are used to tune the mode specific scoring weights and test the completeness of the knowledge bases. When a "stable" software configuration is reached, the set of initial conditions is expanded to 320 by increasing and decreasing the initial separation between the airplanes. This stepwise refinement process provides the large sets of results required to achieve "global" system improvements across the total environment. A subset of the TDG system coded in FORTRAN, DMS-TDG, is currently being tested in the LaRC DMS. DMS-TDG contains a modified version of the SA module, a modified version of the TC module, and the original set of five to nine trial maneuvers. This reduced set of trial maneuvers is used ti insure real-time performance in the DMS. The SA and TC modules were modified to increase the efficiency of the FORTRAN version, but contain the same basic rules as the KBS version used by TDG. The development of DMS-TDG has made it possible to evaluate the TDG against human pilots in a realistic air combat environment. This capability has allowed experienced pilots to interact with the system and comment on its performance and suggest improvements. The pilots comments and suggestions are then incorporated in the lab version for testing and refinement before being included in the DMS version. To date, the TDG has outperformed AML', both in the lab, (fig. 13), and in the DMS against test pilots. 


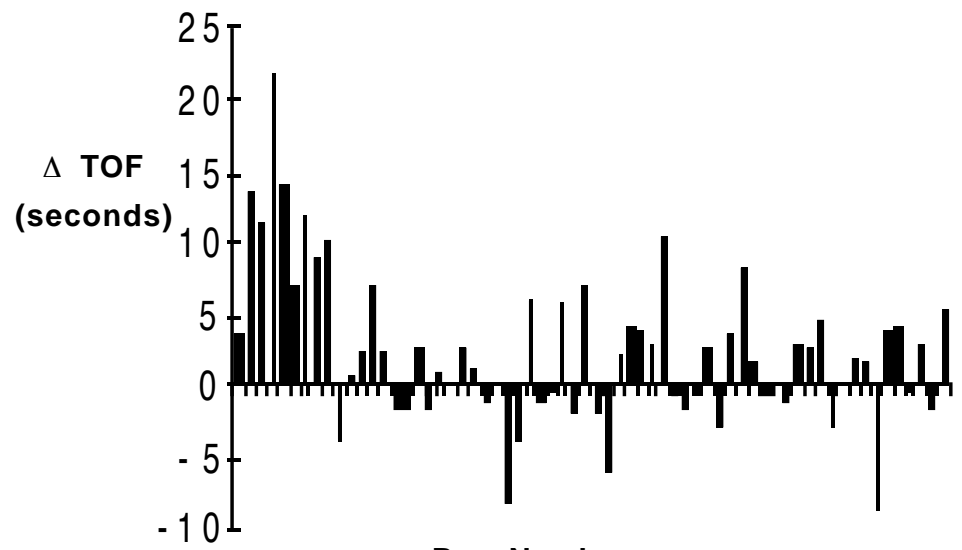

Run Number.

\section{Figure 13. $\triangle$ TOF FOR SET OF 64 ENGAGEMENTS.}

\section{TEST RESULTS}

A set of nine engagements presented in [Eidetics 89] were used to compare the performance of the TDG system with the performance of the AML' in the lab, and against pilots in the DMS. AML' was used as the A airplane in both sets of lab test engagements, and the human pilot flew the A airplane during the DMS runs. Airplanes with identical performance characteristics were used in both the DMS and the lab. The set of nine initial initial conditions, fig.13, favor the A airplane.

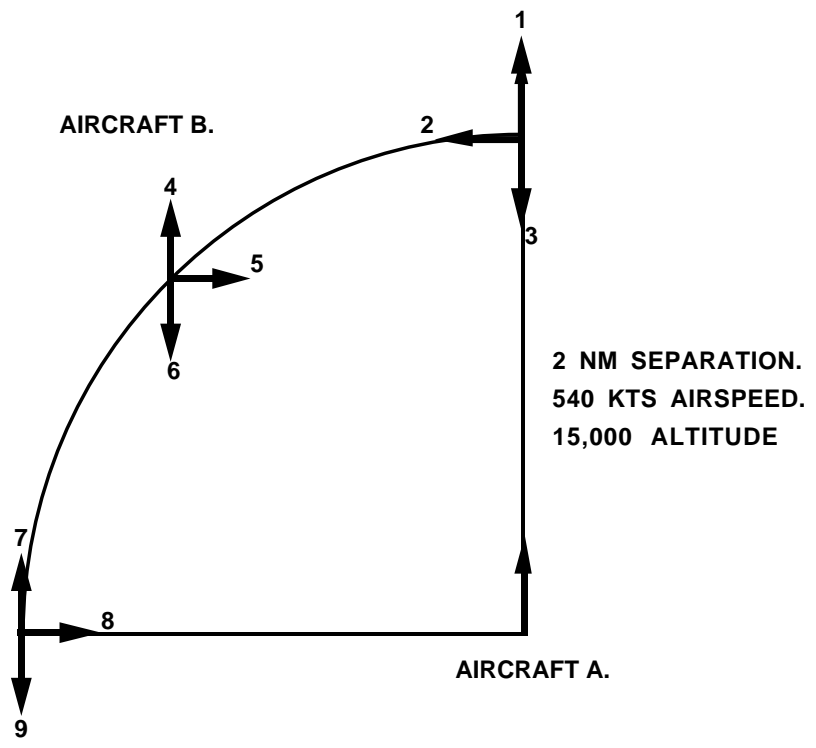

Figure 14. EIDETICS INITIAL CONDITIONS. 
The $\mathbf{B}$ airplane has five neutral starting positions, runs 3, 5, 6, 7, and 9; one offensive starting position, run 8; and 3 defensive starting positions, runs 1, 2, and 4 . There is a 2-nautical mile separation between the opponents and each airplane is at an initial altitude of 15,000 feet and an initial airspeed of 540 knots. All of the engagements were run for 60 seconds. The scoring metric used was an Overall Exchange Ratio (OER), defined as the \# of $\mathbf{A}$ killed / the \# of $\mathbf{B}$ killed. The Eidetics study was conducted using a modified version of the AASPEM program and produced an OER of $\approx 0.72$. The OER was less than 1.0 due to the use of a nonsymmetric set of initial conditions. In the first set of engagements the AML' program was flown against itself and the produced an OER of 0.75 .

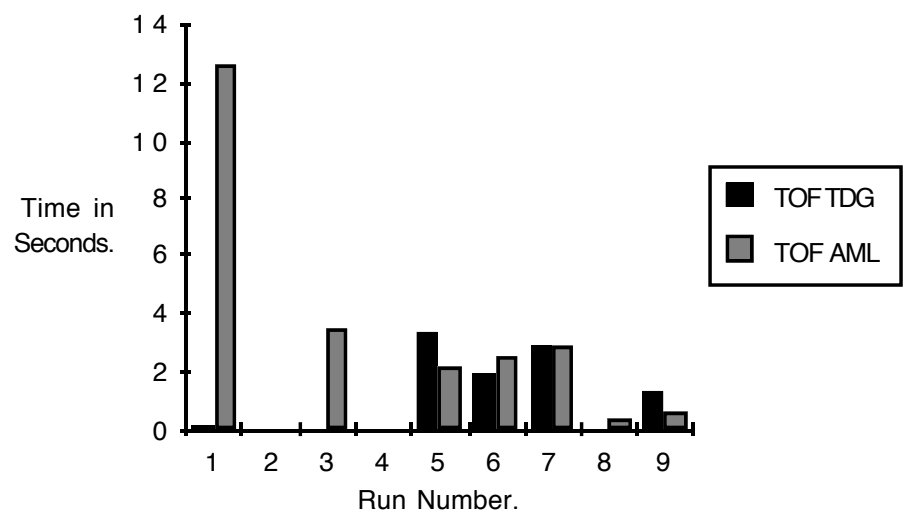

Figure 15. $A M L^{\prime}$ vs $A M L^{\prime}$ TOF.

In the second set of engagements the TDG was used to control the $\mathbf{B}$ airplane and achieved an OER of 1.50, a 100 percent improvement. The test results, (figs. 14, 15), clearly show the superior performance of the TDG system. It is also interesting to note that the maximum OER Eidetics achieved by modifying aircraft performance characteristics was $\approx 0.85$ [Eidetics 1989].

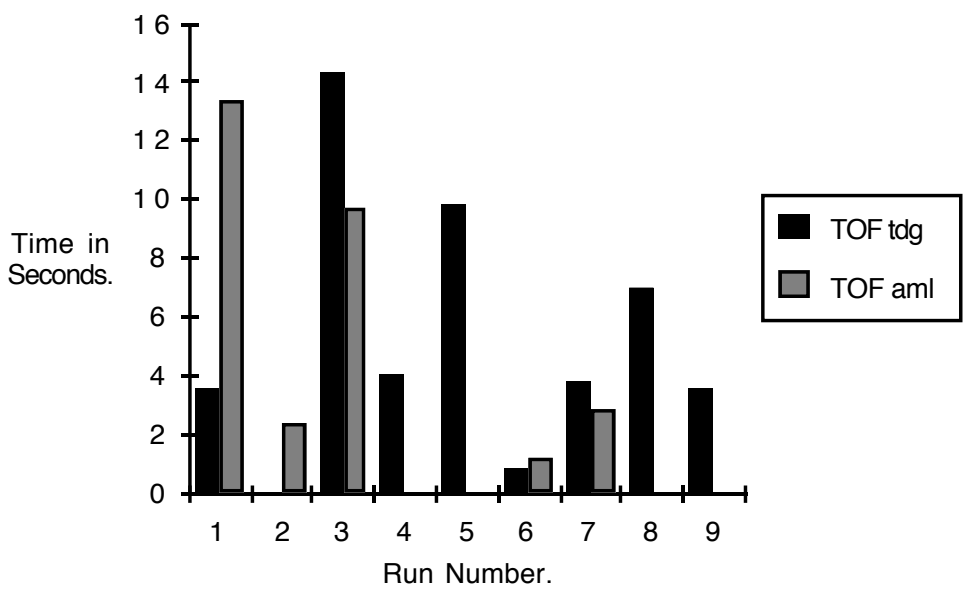




\section{Figure 16. TDG vs $A M L^{\prime}$ TOF.}

The DMS runs were conducted using the research pilot with the most DMS flight time against the TDG-DMS as the opponent. The pilot flew against the set of initial conditions three times, providing a total of 27 runs. TOF data for the DMS runs is not available at this time. The OER for the set of 27 runs was 0.83. As stated earlier, studies done in the lab have shown that the reduced set of trial maneuvers used by DMS-TDG cannot fine track an opponent as effectively as the expanded set used by the TDG. The reduced set of trial maneuvers used by DMS-TDG may account for most of the performance difference between the TDG and DMSTDG.

\section{FUTURE WORK}

Several enhancements to the existing TDG system are planned. The maneuver selection logic will be expanded to replace the use of the trial maneuvers for modes of operation where conventional guidance algorithms provide better performance. This change to the logic and selection module will improve the TDG's ability to track its opponent. Initial lab results have shown that the development of mode-specific maneuver sets will increase system efficiency by reducing the number of maneuvers evaluated for some MO's. The development of logic for two-vs-one engagements is underway. The third aircraft will be dynamically allocated to either the TDG or the opponent at the start of each run. This feature will allow researchers to evaluate the TDG in both two-vs-one and one-vs-two engagements. A system for connecting the Symbolics workstation directly to the DMS real-time computing facilities is also being investigated. The development of such a link would allow the full TDG system to be tested in the DMS against human pilots.

The TGRES system presents an excellent opportunity to evaluate the use of AI programming techniques and knowledge-based systems in a real-time environment. It also clearly shows that the maneuver selection and scoring techniques developed in the late 1960's and early 1970's cannot perform well in the modern tactical environment and are not well suited for evaluating agile aircraft. Figure 16 shows many of the changes in the tactical and simulation environments since the original AML tactical decision generation logic was developed. The use of KBS and AI programming techniques in developing the TDG has allowed a complex tactical decision generation system to be developed that addresses the modern combat environment and agile aircraft in a clear and concise manner. 


$\begin{array}{ll}1968 & 1989 \\ \text { HEAT SEEKING WEAPONS } & \text { ALL-ASPECT WEAPONS DOMINATE } \\ \text { DOMINATE TACTICAL } & \text { TACTICAL SITUATION. (LONGER } \\ \text { SITUATION } & \text { RANGE, FIRE AND FORGET,....) } \\ \text { LIMITED COMPUTING AND } & \text { BETTER COMPUTING AND } \\ \text { MODELING RESOURCES. } & \text { MODELING RESOURCES. } \\ \text { SHORT-RANGE RADAR. } & \text { LONG-RANGE RADAR } \\ \text { SHORT-RANGE WEAPONS. } & \text { LONG-RANGE WEAPONS. } \\ 1 \text { vs 1 } & \text { 2 vs } 1, \text { M vS N } \\ & \text { SUPERMANEUVERABLE AIRCRAFT, } \\ & \text { POINT AND SHOOT CAPABILITY }\end{array}$

\section{Figure 17. 1968 AML vs 1989 TDG.}

\section{CONCLUDING REMARKS}

A KBS TDG is being developed to study WVR air combat engagements. The system incorporates modern airplane simulation techniques, sensors, and weapons systems. The system was developed using several concepts first outlined in the AML program originally developed for use in the LaRC DMS. An updated AML system is being used as a baseline to assess the functional and performance tradeoffs between a conventionally coded system and the AI-based system. Test results have shown that the AI-based TDG system has performed better than AML' in both the TMS and the DMS. The use of a KBS SA module and MO's allows the TDG to more accurately represent the complex decision making process carried out by a pilot. The use of a more extensive set of trial maneuvers and a KBS TC module allows the TDG to fine track the opponent more effectively than AML'. The KBS decision generation logic has proved to be much easier to modify than the AML' FORTRAN source code. The ability to integrate the TDG into the DMS offers a unique opportunity to evaluate the performance of the AI-based TDG software in a real-time tactical environment against human pilots.

\section{REFERENCES}

1. Burgin, G. H. ; et al. : An Adaptive Maneuvering Logic Computer Program for the Simulation of One-on-One Air-to-Air Combat. Vol I and II. NASA CR-2582, CR-2583, 1975.

2. Burgin, G. H. : Improvements to the Adaptive Maneuvering Logic Program. NASA CR3985, 1986.

3. Burgin, G. H. ; and Sidor, L. B. : Rule-Based Air Combat Simulation. NASA CR-4160, 1988.

4. Hankins III, W. W. : Computer-Automated Opponent for Manned Air-to-Air Combat Simulations. NASA TP-1518, 1979. 
5. Kerchner, R. M. ; et al. : The TAC Brawler Air Combat Simulation Analyst Manual (Revision 3. 0). DSA Report \#668.

6. Buttrill, C. S. ; et al. : Draft NASA TM 1989.

7. Taylor, Robert T. ; et al. : Simulated Combat for Advanced Technology Assessments Utilizing The Adaptive Maneuvering Logic Concepts. NASA Order no. L-24468C, Coastal Dynamics Technical Report No. 87-001.

8. $\quad$ McManus, John W. ; Goodrich, Kenneth H. : Draft NASA TM 1989.

9. Goodrich, Kenneth H; McManus John W. :AIAA Paper \#... 